\title{
A PRESENTATION FOR A GROUP OF AUTOMORPHISMS OF A SIMPLICIAL COMPLEX
}

\author{
by M. A. ARMSTRONG
}

(Received 29 May, 1987)

Introduction. The Bass-Serre theorem supplies generators and relations for a group of automorphisms of a tree. Recently K. S. Brown [2] has extended the result to produce a presentation for a group of automorphisms of a simply connected complex, the extra ingredient being relations which come from the 2-cells of the complex. Suppose $G$ is the group, $K$ the complex and $L$ the 1-skeleton of $K$. Then an extension of $\pi_{1}(L)$ by $G$ acts on the universal covering space of $L$ (which is of course a tree) and Brown's technique is to apply the work of Bass and Serre to this action. Our aim is to give a direct elementary proof of Brown's theorem which makes no use of covering spaces, deals with the Bass-Serre theorem as a special case and clarifies the roles played by the various generators and relations.

1. Preliminaries. By a group of automorphisms of a simplicial complex $K$ we mean a group of homeomorphisms of the underlying polyhedron $|K|$ whose elements permute the simplexes of $K$.

A directed edge $e$ of $K$ is a physical edge plus a choice of one of its vertices as initial vertex $i(e)$. The second vertex is then written $t(e)$ and called the terminal vertex. Making the other choice for $i(e)$ produces the reverse $\bar{e}$ of $e$. From now on we refer to directed edges simply as edges. Let $V$ denote the set of vertices and $E$ the set of edges of $K$. These two sets together with the functions

$$
\begin{array}{ll}
E \rightarrow E, & e \rightarrow \bar{e} \\
E \rightarrow V \times V, & e \rightarrow(i(e), t(e))
\end{array}
$$

form a graph $X$ in the sense of Serre [3] because we clearly have $\overline{\bar{e}}=e, \bar{e} \neq e$ and $i(\bar{e})=t(e)$ for each $e \in E$.

If $G$ is a group of automorphisms of $K$ we have a natural action of $G$ on $V$ and on $E$ such that

$$
g i(e)=i(g e) \text { and } g t(e)=t(g e)
$$

for each $g \in G$ and $e \in E$. We shall assume that edges of $K$ are never reversed by the action of $G$. More formally, if $g \in G$ and $e \in E$ then $g e \neq \bar{e}$. Thus the quotient $X / G$ has the structure of a graph. Because $X$ comes from a simplicial complex the initial and terminal vertices of an edge are always different. Of course this property may well be lost on passage to $X / G$.

A path in $K$ (and in $X$ ) joining vertex $u$ to vertex $v$ is an ordered string of edges $e_{1} e_{2} \ldots e_{n}$ such that $i\left(e_{1}\right)=u, i\left(e_{k+1}\right)=t\left(e_{k}\right)$ for $1 \leqslant k \leqslant n-1$, and $t\left(e_{n}\right)=v$. A path of

Glasgow Math. J. 30 (1988) 331-337. 
the form $e \bar{e}$ will be called a round trip. Let $e_{1} \ldots e_{k} e_{k+1} \ldots e_{n}$ be a path and suppose $i\left(e_{k}\right), t\left(e_{k}\right), t\left(e_{k+1}\right)$ are the three vertices of a triangle of $K$. Let $e$ be the edge of this triangle determined by $i(e)=i\left(e_{k}\right), t(e)=t\left(e_{k+1}\right)$. Replacing the pair $e_{k} e_{k+1}$ by $e$ in our original path is called taking a short cut.

We shall work with a complex which is both connected and simply connected. Each of these notions has a combinatorial description. A complex is connected if any two distinct vertices can be joined by a path. It is simply connected if two paths which join the same pair of vertices $u, v$ are always homotopic. That is to say we can convert one path into the other (keeping a path from $u$ to $v$ throughout) by a finite number of steps each of which involves the addition or removal of either a round trip or a short cut.

We adopt the usual notation whereby $G_{u}$ denotes the stabilizer of the vertex $u$. If $g \in G$ happens to fix $u$ we write $g_{u}$ for the element $g$ thought of as a member of $G_{u}$. Of course $G_{e}$ denotes the stabilizer of the edge $e$. If $u$ is a vertex of $e$ then $G_{e}$ is a subgroup of $G_{u}$.

Recall that a graph is a tree if any two of its vertices may be joined by a path, and any path which joins a vertex to itself must contain a round trip.

With $G, K, X$ as above choose a maximal tree $M$ in $X / G$ and lift it [3, Proposition 1.14] to a subtree $T$ of $X$. The vertices of $T$ form a set of representatives for the action of $G$ on the vertices of $X$. For each pair of edges $f, \bar{f}$ from $X / G-M$, select one, say $f$, and lift it to an edge $e$ of $X$ which has its initial vertex $x$ in $T$. Exactly one vertex $z$ of $T$ lies in the same orbit as $t(e)$ and we choose an element $\gamma_{f}$ from $G$ that maps $z$ onto $t(e)$. We can now lift $\bar{f}$ to $\left(\gamma_{f}\right)^{-1} \bar{e}$. This has its initial vertex $z$ in $T$ and $\gamma_{f}^{-}=\left(\gamma_{f}\right)^{-1}$ sends the vertex $x$ of $T$ to its terminal vertex (Fig. 1). Finally we extend the correspondence $f \rightarrow \gamma_{f}$ over the edges of $M$ by setting $\gamma_{f}=1$ (the identity element of $G$ ) whenever $f \in M$.
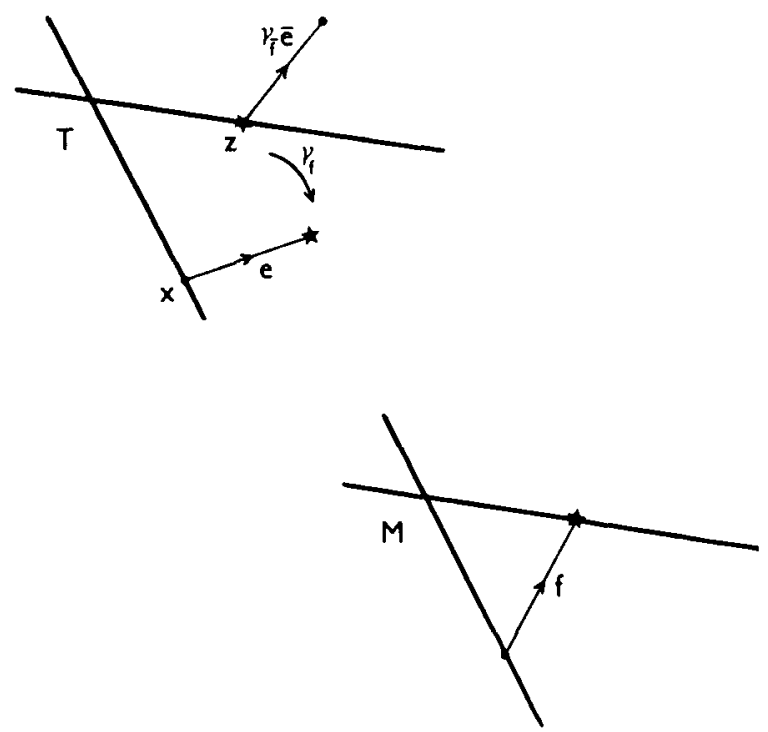

Figure 1 
The elements of all the $G_{w}$, where $w$ is a vertex of $T$, and the $\gamma_{f}$, where $f$ is an edge of $X / G$, together generate $G$. They satisfy the relation

$$
\gamma_{\bar{f}} g_{x} \gamma_{f}=\left(\gamma_{\bar{f}} g \gamma_{f}\right)_{z}
$$

whenever $g$ fixes the canonical lift $e$ of $f$, plus relations which come from the triangles of $K$ and which will be described in the next section.

2. Tail wagging. Let $g \in G$ and let $e_{1} e_{2} \ldots e_{n}$ be a path which joins a vertex $v$ of $T$ to $g v$. If the path lies entirely in $T$ then $g v=v$ because no two vertices of $T$ lie in the same orbit. Therefore $g=g_{v}$, where as usual $g_{v}$ denotes the element $g$ interpreted as a member of $G_{v}$. Otherwise there is a first edge $e_{m}$ that is not in $T$. The path $e_{m} e_{m+1} \ldots e_{n}$ will be called the tail of $e_{1} e_{2} \ldots e_{n}$. Let $x_{1}$ be the initial vertex of $e_{m}$. Project $e_{m}$ into $X / G$ to give an edge $f_{1}$. The canonical lift $e^{1}$ of $f_{1}$ into $X$ has its initial vertex in $T$, so $i\left(e^{1}\right)=x_{1}$. Choose an element $a_{x_{1}} \in G_{x_{1}}$ which sends $e^{1}$ to $e_{m}$. Let

$$
e_{k}^{1}=\left(\gamma_{\bar{f}_{1}} a_{x_{1}}^{-1}\right) e_{k}
$$

for $m+1 \leqslant k \leqslant n$ and replace $e_{1} e_{2} \ldots e_{n}$ by the new path $e_{m+1}^{1} e_{m+2}^{1} \ldots e_{n}^{1}$. We call this process tail wagging. Our new path begins at

$$
z_{1}=t\left(\gamma_{\bar{f}_{1}} e^{1}\right)=i\left(e_{m+1}^{1}\right)
$$

which is a vertex of $T$ and ends at $\left(\gamma_{\bar{f}_{1}} a_{x_{1}}^{-1} g\right) v$; see Fig. 2 . We walk along it to the first point $x_{2}$ where it quits $T$ and repeat the above procedure. Since we shorten the tail at each step we eventually obtain a path which lies entirely in $T$ and ends at, say,

$$
\left(\gamma_{\bar{f}_{r}} a_{x_{r}}^{-1} \ldots \gamma_{\bar{f}_{2}} a_{x_{2}}^{-1} \gamma_{\bar{f}_{1}} a_{x_{1}}^{-1} g\right) v \text {. }
$$

Then $\gamma_{\bar{f}_{r}} a_{x_{r}}^{-1} \ldots \gamma_{\bar{f}_{1}} a_{x_{1}}^{-1} g$ must fix $v$, say $\gamma_{\bar{f}_{r}} a_{x_{r}}^{-1} \ldots \gamma_{\bar{f}_{1}} a_{x_{1}}^{-1} g=a_{v} \in G_{v}$. We now have

$$
g=a_{x_{1}} \gamma_{f_{1}} \ldots a_{x_{r}} \gamma_{f_{r}} a_{v}
$$

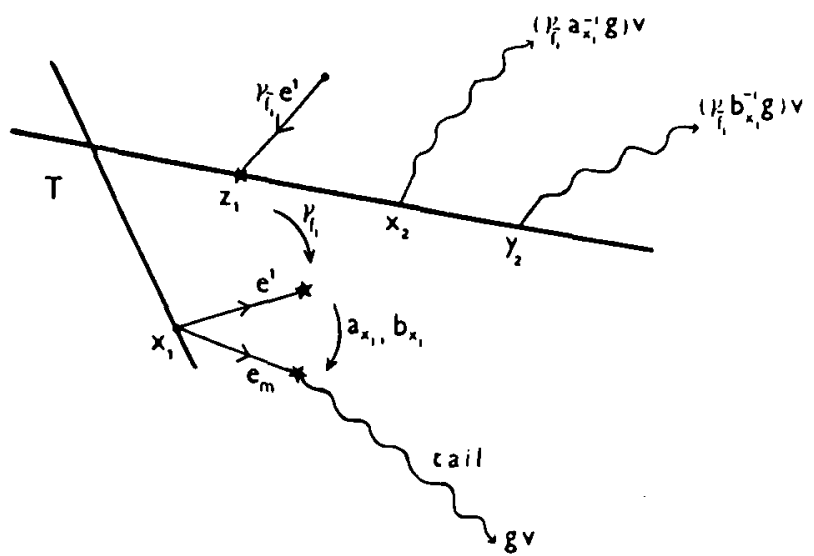

Figure 2 
This shows that the elements of the $G_{w}, w \in T$, together with the $\gamma_{f}, f$ an edge of $X / G$, do indeed generate $G$.

Now for the extra relations mentioned in $\$ 1$. Our group $G$ acts on the collection of all triangles in $K$. From each orbit we choose a triangle which has a vertex in $T$. Let $\Delta$ be such a triangle and let $v$ be a vertex of $\Delta$ which belongs to $T$. Walking round the boundary of $\Delta$ (there is a choice of direction here) produces a path which joins $v$ to $v=1 v$, and tail wagging this path expresses the identity element of $G$ as a word $r_{\Delta}$ in our generators. The missing relations have the form

$$
r_{\Delta}=1 \text {, }
$$

one for each orbit of triangles in $K$.

With the notation established above let $* G_{w}$ denote the free product of the stabilizers of the vertices of $T$, and $F$ the free group generated by symbols $\lambda_{f}$, one for each edge of $X / G$. Let $R$ be the normal consequence in $\left(* G_{w}\right) * F$ of the words

$$
\begin{gathered}
\lambda_{f}(f \text { an edge of } M), \\
\left.\lambda_{\bar{f}} \lambda_{f} \text { (for each edge of } X / G\right), \\
\lambda_{\bar{f}} g_{x} \lambda_{f}\left(\gamma_{\bar{f}} g \gamma_{f}\right)_{z}^{-1} \text { (when } g \text { fixes the canonical lift } e \text { of } f \text { ), and } \\
r_{\Delta}^{\lambda} \text { (obtained from } r_{\Delta} \text { by changing each occurrence of } \gamma_{f} \\
\text { to } \lambda_{f} \text {, one such for each orbit of triangles). }
\end{gathered}
$$

Presentation Theorem. If $G$ is a group of automorphisms of a connected simply connected complex $K$, and if no edge of $K$ is reversed by the action of $G$, then $G$ is isomorphic to $\left[\left(* G_{w}\right) * F\right] / R$.

If $K$ is one-dimensional, so that $X$ is a tree, this is the Bass-Serre Theorem [3, Theorème I.13]. For dimension two or more the extra relations were provided by K. S. Brown [2].

3. Homotopy. We shall produce an isomorphism

$$
\psi: G \rightarrow\left[\left(* G_{w}\right) * F\right] / R
$$

as follows. Take a vertex $v$ of $T$ as base point. Given $g \in G$, choose a path $\alpha$ in $K$ which joins $v$ to $g v$. By tail wagging $\alpha$ we express $g$ as a word $a_{x_{1}} \gamma_{f_{1}} \ldots a_{x_{r}} \gamma_{f_{r}} a_{v}$ and we define

$$
\psi(g)=a_{x_{1}} \lambda_{f_{1}} \ldots a_{x_{r}} \lambda_{f_{r}} a_{v} R .
$$

Of course various choices are involved here and we must show that $\psi$ is well defined. For a particular path $\alpha$ joining $v$ to $g v$ the first ambiguity occurs in the choice of the element $a_{x_{1}} \in G_{x_{1}}$ which maps $e^{1}$ to $e_{m}$. That a different choice at this and subsequent stages gives the same coset for $\psi(g)$ is verified exactly as in [1]. As to the choice of the actual path $\alpha$ we need only check that altering $\alpha$ by the addition or removal of a single round trip or short cut makes no difference to the value of $\psi(g)$. 
Let $\alpha$ be the string $e_{1} e_{2} \ldots e_{n}$ leading to the word $a_{x_{1}} \lambda_{f_{1}} \ldots a_{x_{r}} \lambda_{f_{r}} a_{v}$ in $\left(* G_{w}\right) * F$. Suppose we add the round trip $e_{0} \bar{e}_{0}$. Let $f$ be the image of $e_{0}$ in $X / G$ and $e$ the canonical lift of $f$ back into $X$, so that $i(e)=x$ lies in $T$. Tail wagging the new path gives a word of the form

$$
a_{x_{1}} \lambda_{f_{1}} \ldots a_{x_{k}} \lambda_{f_{k}}\left(a_{x} \lambda_{f}\right)\left(\lambda_{\bar{f}}\right)\left(a_{x}^{-1}\right) a_{x_{k+1}} \lambda_{f_{k+1}} \ldots a_{x_{r}} \lambda_{f_{r}} a_{v}
$$

if $x$ is distinct from all the $x_{i}$, or possibly

$$
a_{x_{1}} \lambda_{f_{1}} \ldots a_{x_{k}} \lambda_{f_{k}}\left(a_{x}^{\prime} \lambda_{f}\right)\left(\lambda_{\bar{f}}\right)\left(a_{x}^{\prime-1} a_{x_{k+1}} \lambda_{f_{k+1}}\right) \ldots a_{x_{r}} \lambda_{f_{r}} a_{v}
$$

if $x=x_{k+1}$. We have used parentheses to emphasise groups of symbols which correspond to a single tail wag. Removing a round trip has the opposite effect and clearly neither process changes the coset of the original word.

Suppose now we start with $\alpha$ and take a short cut, calling the new path $\beta$. Tail wagging $\beta$ proceeds as for $\alpha$ until we reach a vertex $w$ of $T$ from which the modified $\alpha$ runs along two sides of a triangle $\Delta_{0}$, whereas what remains of $\beta$ short cuts along the third side. Clearly $\alpha$ and $\beta$ give the same $R$-coset if $r_{\Delta_{0}}^{\lambda}$ belongs to $R$. The following lemma shows that this is the case.

LeMma. Let $\Delta$ be a triangle which has a vertex $u$ in $T$ and suppose that $r_{\Delta}^{\lambda} \in R$. If $g$ sends $\Delta$ to a triangle $\Delta_{0}$ which also has a vertex in $T$ then $r_{\Delta_{0}}^{\lambda} \in R$.

Proof. We may as well assume that the situation is represented by Fig. 3, the only possible ambiguity being the direction in which we choose to go round one or other of the triangles. As is easily checked a reversal of direction produces a word which is $R$-equivalent to the inverse of the original word, and does not affect our lemma.

For $m=1,2,3$ let $f_{m}$ be the projection of $e_{m}$ into $X / G$ and $e^{m}$ the canonical lift of $f_{m}$ back into $X$ with $i\left(e^{m}\right) \in T$. Tail wagging $e_{1} e_{2} e_{3}$ gives, say,

$$
r_{\Delta}^{\lambda}=a_{u} \lambda_{f_{1}} a_{x_{2}} \lambda_{f_{2}} a_{w} \lambda_{f_{3}} b_{u}
$$

and taking the boundary of $\Delta_{0}$ in the direction shown leads to

$$
r_{\Delta_{0}}^{\lambda}=\left(a_{w} \lambda_{f_{3}}\right)\left(b_{u} c_{u} a_{u} \lambda_{f_{1}}\right)\left(a_{x_{2}} \lambda_{f_{2}}\right) d_{w}
$$

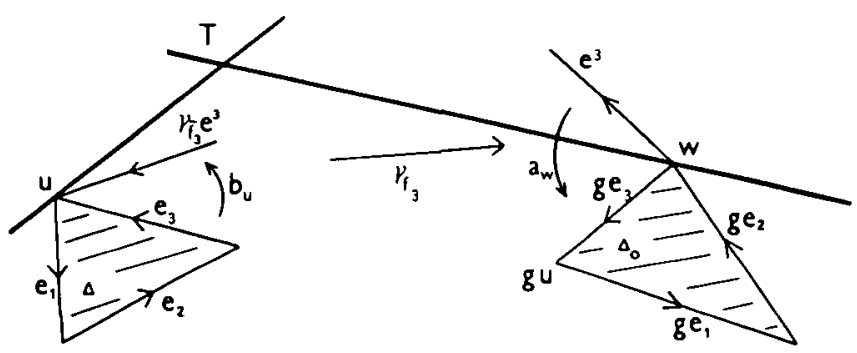

Figure 3 
where $c_{u}$ fixes the edge $e_{3}$. Now

$$
\begin{aligned}
d_{w} & =\left[\left(a_{w} \gamma_{f_{3}} b_{u} c_{u} a_{u} \gamma_{f_{1}} a_{x_{2}} \gamma_{f_{2}}\right)^{-1}\right]_{w} \\
& =\left[\left(a_{u} \gamma_{f_{1}} a_{x_{2}} \gamma_{f_{2}}\right)^{-1}\left(a_{w} \gamma_{f_{3}} b_{u} c_{u}\right)^{-1}\right]_{w} \\
& =\left[\left(a_{w} \gamma_{f_{3}} b_{u}\right)\left(a_{w} \gamma_{f_{3}} b_{u} c_{u}\right)^{-1}\right]_{w} \quad \text { by } \\
& =a_{w}\left(\gamma_{f_{3}} b_{u} c_{u}^{-1} b_{u}^{-1} \gamma_{f_{3}}\right)_{w} a_{w}^{-1} \\
& =a_{w} \lambda_{f_{3}} b_{u} c_{u}^{-1} b_{u}^{-1} \lambda_{\bar{f}_{3}} a_{w}^{-1}
\end{aligned}
$$

because $b_{u} c_{u} b_{u}^{-1}$ fixes $\gamma_{\gamma_{3}} \bar{e}^{-3}$. Substitution in (2) gives

$$
r_{\Delta_{0}}^{\lambda}=\left(a_{w} \lambda_{f_{3}} b_{u} c_{u}\right) r_{\Delta}^{\lambda}\left(a_{w} \lambda_{f_{3}} b_{u} c_{u}\right)^{-1}
$$

and shows that $r_{\Delta_{0}}^{\lambda}$ belongs to $R$.

4. Composite paths. To complete the proof of the Presentation Theorem we show that $\psi$ is a homomorphism and a bijection.

Given elements $g, h \in G$ join $v$ to $g v$ and $h v$ by paths $\alpha, \beta$ respectively. Tail wag $\alpha$ and $\beta$ to give say

$$
\begin{aligned}
& \psi(g)=a_{x_{1}} \lambda_{f_{1}} \ldots a_{x_{r}} \lambda_{f^{\prime}} a_{v} R, \\
& \psi(h)=b_{x_{1}} \lambda_{f_{1}} \ldots b_{x_{s}^{\prime}} \lambda_{f_{s}} b_{v} R .
\end{aligned}
$$

Now $\alpha$ followed by the image of $\beta$ under $g$ joins $v$ to $(g h) v$. Using this composite path to compute $\psi(g h)$ gives

$$
\psi(g h)=\left(a_{x_{1}} \lambda_{f_{1}}\right) \ldots\left(a_{x_{r}} \lambda_{f_{r}}\right)\left(a_{v}\right)\left(b_{x_{1}^{\prime}} \lambda_{f_{1}^{\prime}}\right) \ldots\left(b_{x_{s}^{\prime}} \lambda_{f^{\prime}}\right) b_{v} R
$$

provided $x_{1}^{\prime}$ is not the same as $v$, or

$$
\psi(g h)=\left(a_{x_{1}} \lambda_{f_{1}}\right) \ldots\left(a_{x_{r}} \lambda_{f_{r}}\right)\left(a_{v} b_{x_{1}} \lambda_{f_{1}^{\prime}}\right) \ldots\left(b_{x_{s}^{\prime}} \lambda_{f_{s}^{\prime}}\right) b_{v} R
$$

if $x_{1}^{\prime}=\boldsymbol{v}$. Therefore $\psi(g h)=\psi(g) \psi(h)$ and $\psi$ is a homomorphism.

Our construction of $\psi$ ensures that if $\psi(g)=R$ then $g=1$. So $\psi$ is injective. The cosets $h_{w} R$ ( $w$ a vertex of $T$ and $\left.h w=w\right)$ and $\lambda_{f} R(f$ an edge of $X / G)$ together generate $\left[\left(* G_{w}\right) * F\right] / R$. We show $\psi$ surjective by checking that $\psi(h)=h_{w} R$ and $\psi\left(\gamma_{f}\right)=\lambda_{f} R$. If $x, y$ are vertices of $T$ write $\overrightarrow{x y}$ for the geodesic (shortest path) in $T$ which joins $x$ to $y$. This geodesic is unique because $T$ is a tree. Suppose $h$ fixes the vertex $w$ of $T$. Let $x$ be the closest vertex of $T$ to $v$ such that $\overrightarrow{x w}$ is left fixed by $h$, and note that $\overrightarrow{v x}$ followed by the image of $\overrightarrow{x v}$ under $h$ joins $v$ to $h v$. This path leaves $T$ for the first time at $x$ and a single tail wag using $h_{x}^{-1}$ shows that $\psi(h)=h_{x} R$. But $h$ fixes all of the geodesic $\overrightarrow{x w}$. Hence $h_{x} R=h_{w} R$ as required.

Finally, if $f$ is an edge of $X / G$ with canonical lift $e$ in $X$ then (with the usual notation) the composite path $\overrightarrow{v x}$ followed by $e$ followed by $\gamma_{f}(\overrightarrow{z v})$ joins $v$ to $\gamma_{f} v$. This path leaves $T$ for the first time at $x$ and a single tail wag by $\gamma_{\bar{f}}$ shows $\psi\left(\gamma_{f}\right)=\lambda_{f} R$. This completes the argument. 


\section{REFERENCES}

1. M. A. Armstrong, Trees, tail wagging and group presentations, L'Enseignement Mathematique 32 (1986), 261-270.

2. K. S. Brown, Presentations for groups acting on simply connected complexes, J. Pure and Applied Algebra 32 (1984), 1-10.

3. J.-P. Serre, Arbres, Amalgames, $S L_{2}$, Astérisque 46 (Soc. Math. de France 1977).

Department of Mathematical Sciences

SCIENCE LABORATORIES

SOUTH ROAD

DURHAM DH1 3LE

ENGLAND 